\section{Prevalência e fatores determinantes do consumo de medicamentos no Município de Fortaleza, Ceará, Brasil}

\author{
Prevalence and determinants of medicines \\ consumption in Fortaleza, Ceará, Brazil
}

\begin{abstract}
The objective of this work was to investigate the prevalence and determinants of medicines consumption in Fortaleza, Ceará, Brazil. A population-based cross-sectional study was carried out in a representative sample of 331 households. A total of 1,370 persons living in Fortaleza were interviewed from October 2002 to January 2003, and 1,366 were selected for this study. Consumers were defined as all persons having used one or more drugs during the preceding 15 days. The prevalence of medicine consumption was $49.7 \%$. The following were the predictive factors for medicines consumption according to the multivariate logistic regression analysis: family income $>3$ minimum wages, older age $(\geq 50)$, female gender, $\geq 3$ persons living in household, chronic disease, having a health insurance plan, and one or more visits to the doctor in the last three months. Prevalence of medication use was high in Fortaleza but was similar to that found elsewhere in Brazil and in other countries. Inequality in the access of medications was identified.
\end{abstract}

Drug Use Habits; Drug Utilization; Pharmacoepidemiology
Paulo Sérgio Dourado Arrais 1,2 Luciara Leite Brito 2 Maurício Lima Barreto 2 Helena Lutéscia L. Coelho 1,2
O medicamento é um bem essencial à saúde e uma importante ferramenta terapêutica nas mãos dos médicos, sendo responsável por parte significativa da melhoria da qualidade e expectativa de vida da população. Entretanto, seu uso irracional e suas conseqüências elevam os gastos na área da saúde, o que torna o tema de grande relevância para os que trabalham com saúde pública.

A tendência de crescimento do consumo de medicamentos nos países desenvolvidos e subdesenvolvidos, influenciada, em parte, pelo aumento da expectativa de vida das populações e o conseqüente aumento dos gastos na área da saúde, aliada às restrições orçamentárias do setor público, tem levado os governantes a promoverem, em seus países, reformas no setor. No Brasil, este problema vem prejudicando a consolidação do Sistema Único de Saúde (SUS). Apesar da nítida melhora do sistema, desde sua implementação, ainda existem dificuldades de acesso às unidades de atenção básica e aos medicamentos, e são inúmeras as reclamações sobre a qualidade dos serviços 1,2. Conseqüentemente, as pessoas têm sido obrigadas a pagar planos de saúde e a comprar seus medicamentos 1,3,4. Em alguns casos, o paciente fica sem o tratamento por não ter condições financeiras para comprá-lo 4,5.

Para a Organização Mundial da Saúde 6 (p. 4), o uso racional de medicamentos ocorre quan- 
do "os pacientes recebem o medicamento apropriado às suas necessidades clínicas, nas doses $e$ posologias corretas, por um período de tempo adequado e ao menor custo para eles e para a comunidade". Entretanto, o seu uso pode ser influenciado por diversos fatores 7,8, alguns dizem respeito à oferta de produtos no mercado, seu número, variedade e qualidade, bem como a força da regulação vigente e o preço. O acesso aos serviços de saúde, a cultura médica e a facilidade em adquirir medicamentos também são fatores considerados de grande peso 7,8 . Todos esses aspectos são influenciados pelo marketing direto e indireto da indústria farmacêutica, que induz comportamentos, necessidades e os mais variados interesses 9 .

Estudos de base populacional, realizados em vários países, também mostram que a utilização de medicamentos está influenciada, entre outros, pelo sexo $1,10,11,12,13,14,15,16,17,18,19,20$, idade $1,10,11,12,13,14,15,16,17,18,19,20,21$, grau de escolaridade $13,17,21$, renda familiar mensal 1,4 , classe social 4,17 , ocupação $13,17,21$, número de residentes no domicílio 11,13, número de consultas médicas 4,11,16, autopercepção do estado de saúde 10,12, cuidados com a saúde e padecer de doença crônica 16, e consulta à farmácia nos últimos 12 meses 11 .

No Brasil, a maior parte desses estudos foi desenvolvida no Sul e Sudeste do país 10,11,14,18, 19,20. As diferenças de indicadores sócio-econômicos, epidemiológicos e de acesso e utilização de serviços de saúde existentes entre essas regiões e o Nordeste do Brasil 2, onde está localizado o Município de Fortaleza, justifica esperar-se encontrar peculiaridades no consumo de medicamentos.

Conhecer o perfil epidemiológico e os fatores que influenciam o consumo dos medicamentos na comunidade tem sido um instrumento importante para o delineamento de medidas de contenção de gastos farmacêuticos e melhoria das políticas de assistência à saúde do cidadão.

O presente trabalho tem por finalidade determinar a prevalência e os determinantes do consumo de medicamentos pela população de Fortaleza, focalizando variáveis demográficas, sócio-econômicas, biológicas e de indicadores da condição de saúde e de utilização de serviços de saúde.

\section{Métodos}

Trata-se de um estudo de delineamento transversal de base populacional, realizado no $\mathrm{Mu}$ nicípio de Fortaleza, Ceará, no período de ou- tubro de 2002 a janeiro de 2003. A unidade de observação foi o indivíduo no domicílio.

Fortaleza sofre com o aumento acelerado da industrialização urbana e conseqüente adensamento populacional. O município contou no Censo Demográfico 2000 com 2.141.402 habitantes 22, correspondendo a um terço da população do estado. O desemprego entre a população gira em torno dos 12,0\%. Existe grande desigualdade social, onde $20,0 \%$ da população concentra $80,0 \%$ das riquezas e $37,0 \%$ possuem renda igual ou menor que meio salário mínimo 23. A municipalização dos serviços de saúde vem ocorrendo desde 1991. Entretanto, essas mudanças e transformações acontecem em um momento de crise do setor saúde, onde ocorre o aumento da demanda e gastos financeiros, aliadas às restrições orçamentárias do setor público. As dificuldades enfrentadas dificultam a consolidação do SUS no município.

A amostra para o estudo foi calculada em função do número de domicílios de Fortaleza $(n=526.079) 22$, de forma a garantir a representatividade da área territorial em que a pesquisa foi realizada. O plano de amostragem foi elaborado com base em técnicas de processos probabilísticos com estratificação dos bairros e setores censitários de Fortaleza.

Para o dimensionamento da amostra foi adotado um modelo de amostragem estratificada proporcional com desvio amostral de 5,39\%, um nível de segurança de 95,0\% e uma probabilidade de ocorrência do evento (consumo de medicamentos) fixada em 0,5 , perfazendo o total de 331 domicílios a serem visitados.

A probabilidade de ocorrência do evento foi feita com base em estudos realizados no Brasil, que mostram uma prevalência de uso variando entre $24,0 \%$ e $70,0 \% 11,14,18,19,20$. A partir daí o consumo foi estimado em $50,0 \%$.

Foram selecionados aleatoriamente três bairros entre cada uma das nove regiões administrativas de Fortaleza, e de cada bairro dois setores censitários, ou seja, os 331 domicílios foram selecionados entre esses 54 setores censitários. Em cada setor censitário estabeleceuse que o ponto de partida seria a esquina do quarteirão que se encontrasse mais à esquerda do mapa do setor. O primeiro domicílio a ser visitado foi selecionado a partir do último algarismo correspondente ao número do setor censitário selecionado. Após esta identificação e, seguindo o sentido horário, foram visitados um em cada dez domicílios até completar o número determinado para cada setor. Os domicílios foram substituídos no caso de estarem permanentemente desocupados, ou habitados, porém fechados na ocasião de três visitas segui- 
das, e no caso de recusa formal do chefe da família ou responsável.

As informações foram coletadas por meio de um questionário padronizado, contendo questões de fácil compreensão, e previamente testado. Foram coletados dados demográficos; sócio-econômicos; hábitos sociais; consumo de medicamentos; indicadores de saúde e utilização de serviços de saúde; experiências com reações adversas a medicamentos no período do estudo ou em alguma vez na vida, e suas características; informações sobre a conduta do médico, do dispensador e do paciente no processo da prescrição e dispensação dos medicamentos. As duas últimas não apresentadas no presente trabalho.

Para a aplicação do questionário foram selecionados e treinados dez entrevistadores, acadêmicos do curso de Farmácia, Universidade Federal do Ceará (UFCE), que aplicaram os questionários nos domicílios selecionados.

Para a entrevista individual foi obtido consentimento prévio de todas as pessoas (escrito ou verbal). As informações relativas às pessoas com idade menor que 15 anos e idosos ou adultos com problemas de cognição ou doença foram dadas por seus responsáveis legais. No estudo, 30,0\% foram considerados informantes.

Para o levantamento da renda, decidiu-se montar uma tabela com sete faixas salariais e uma variável sem rendimento, na tentativa de obter o maior número possível de informações a este respeito. Os entrevistadores foram orientados para solicitar que as pessoas apresentassem o(s) medicamento(s) utilizado(s) ou, se fosse o caso, mostrassem a receita médica.

\section{Variáveis do estudo}

No presente estudo, consumo de medicamentos foi considerado a variável dependente. Foram considerados consumidores de medicamentos todas as pessoas que nos 15 dias anteriores à entrevista tinham consumido pelo menos um medicamento. As variáveis independentes coletadas foram: (a) demográficas: número de pessoas residentes no domicílio $(\leq 3 ; 4$ a $5 ; \geq 6$ ), sexo (masculino ou feminino), idade em anos completos (0-15, 16-49, 50-98), estado conjugal (casado, não-casado); (b) sócio-econômicas: escolaridade ( $\leq 8$ anos e $>8$ anos), renda familiar mensal (até 3 salários mínimos e 4 a 20 salários mínimos), ocupação (sim ou não). O salário mínimo na época da realização da pesquisa correspondia a $\mathrm{R} \$ 200,00$ (duzentos Reais); (c) indicadores da condição de saúde: história de doenças crônicas referidas pelo indivíduo (sim ou não), autopercepção do es- tado de saúde; (d) indicadores de utilização de serviços de saúde: internação hospitalar nos últimos 12 meses (sim ou não), consulta médica nos últimos três meses (sim ou não), posse de plano privado de saúde (sim ou não).

Para garantir a qualidade dos dados, reentrevistas parciais, pessoais ou telefônicas foram realizadas com $20,0 \%$ dos participantes.

Os dados foram armazenados no programa Epi Info 6.0, com dupla entrada da informação, e analisados com o auxílio do programa Stata, versão 7.0. O plano de análise dos dados incluiu: a obtenção de freqüências simples das variáveis de interesse; investigações das características das variáveis em estudo associadas ao consumo de medicamentos, utilizando-se o teste qui-quadrado para verificar diferenças de proporções, com nível de significância $\mathrm{p}<0,05$; análise bivariada, empregando como medida de efeito a razão de prevalência e seus respectivos intervalos de confiança; a análise multivariada foi realizada por meio de regressão logística não condicional, obedecendo ao modelo hierárquico proposto na Figura 1.

Os fatores sócio-econômicos, considerados como o principal fator desencadeador da sucessão de eventos que culminaram com o aparecimento de quadros mórbidos e consumo de medicamentos, compõem a primeira etapa da análise. Os fatores demográficos e os relativos à situação de saúde e utilização de serviços compõem, respectivamente, a segunda e terceira etapas. A quarta etapa da análise é o bloco da avaliação subjetiva (autopercepção do estado de saúde).

A inclusão de cada variável dependeu da significância estatística aferida pela razão de prevalência e seus respectivos intervalos de confiança. No modelo final restaram as variáveis que, acrescidas ao modelo, apresentavam um valor $\mathrm{p}<0,10$ quando ajustadas pelas variáveis dos níveis superiores e do mesmo nível. A razão de prevalência foi obtida aplicando-se o método elaborado por Zhang \& Yu 24, com o auxílio do comando relrisk do programa estatístico Stata. Para todas as análises considerouse o efeito do desenho amostral.

O protocolo da pesquisa foi submetido e aprovado pelo Comitê de Ética em Pesquisa do complexo hospitalar da UFCE.

\section{Resultados}

O estudo incluiu 1.378 indivíduos, sendo que oito $(0,6 \%)$ foram perdas e recusas, resultando em 1.370 pessoas entrevistadas. Dessas, quatro foram excluídas da análise, pois os questioná- 
Figura 1

Modelo hierárquico do processo de determinação do consumo de medicamentos.

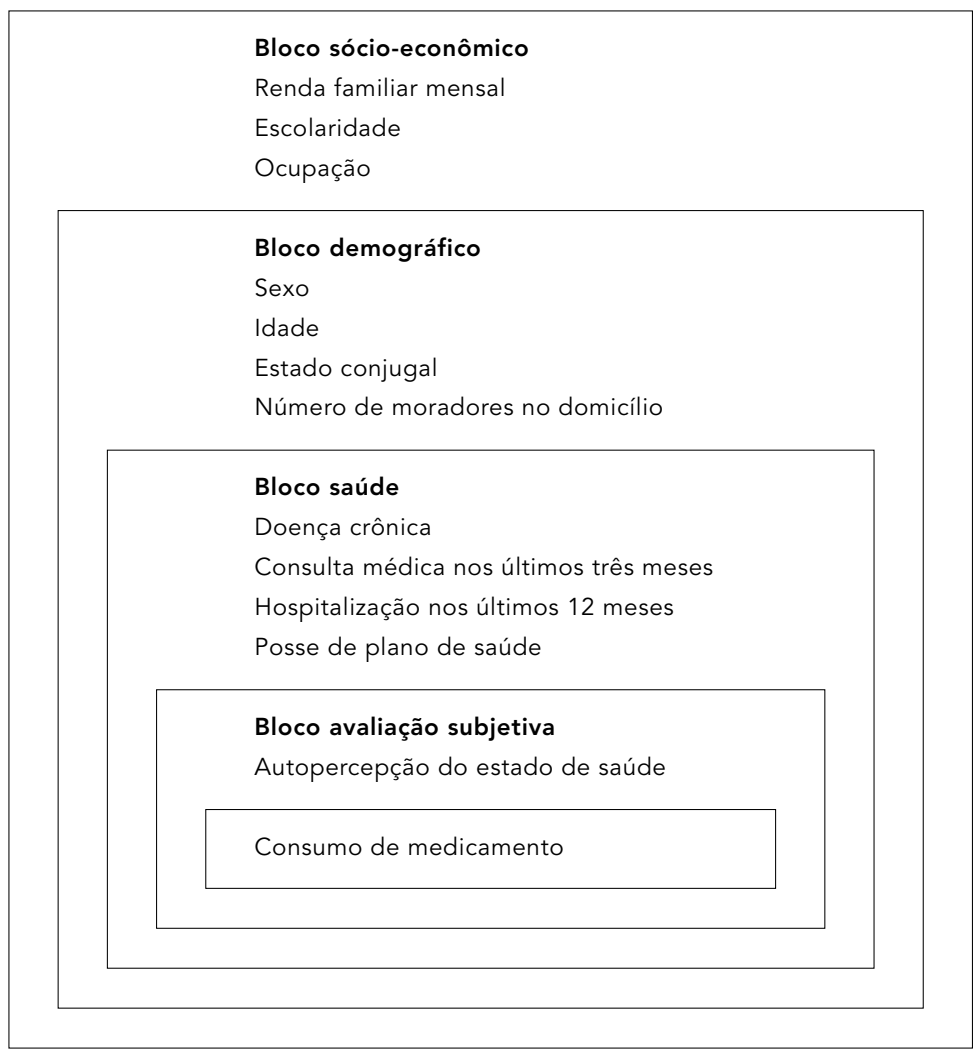

rios estavam incompletos. Da população estudada, 679 pessoas $(49,7 \%)$ referiram ter consumido pelo menos um medicamento nos últimos 15 dias.

Na Tabela 1 observa-se a distribuição dos indivíduos segundo as variáveis estudadas. O consumo foi maior entre as pessoas que pertenciam às famílias cuja renda mensal era superior a três salários mínimos (55,2\%), com oito ou mais anos de escolaridade $(52,7 \%)$, do sexo feminino $(55,6 \%)$, com idade maior ou igual a 50 anos $(67,2 \%)$, casados $(51,0 \%)$, de famílias com três ou menos pessoas no domicílio $(56,0 \%)$, que referiram doença crônica $(88,0 \%)$, que possuíam cobertura por plano de saúde $(66,0 \%)$, que realizaram uma ou mais consultas médicas nos últimos três meses $(64,5 \%)$ ou que tenham se hospitalizado uma ou mais vezes nos últimos 12 meses $(62,0 \%)$ e entre os indivíduos cuja autopercepção do estado de saúde é ruim ou muito ruim (69,9\%). Com relação à ocupação, o consumo foi maior entre os não-ocupados $(47,2 \%)$.

Observou-se diferença estatisticamente significante $(\mathrm{p}<0,05)$ no uso de medicamentos de acordo com a renda familiar, escolaridade, sexo, idade igual ou maior que 50 anos, estado conjugal, número de residentes por domicílio (com três ou menos e quatro ou cinco indivíduos), ter ou não doença crônica, plano de saúde, uma ou mais consultas ou hospitalizações, respectivamente, nos últimos 3 e 12 meses (Tabela 1).

Na análise bivariada (Tabela 2), observouse uma associação positiva e estatisticamente significante entre consumo de medicamentos e as seguintes variáveis: renda familiar mensal maior que três salários mínimos (razão de prevalência - $\mathrm{RP}=1,3$; intervalo de confiança de 95\% - IC95\%: 1,2-1,5), escolaridade maior que oito anos (RP = 1,2; IC95\%: 1,1-1,4), sexo feminino ( $\mathrm{RP}=1,4$; IC95\%: 1,3-1,5), idade entre $50 \mathrm{e}$ 98 anos (RP = 1,7; IC95\%: 1,4-1,9), domicílios com três ou menos pessoas $(\mathrm{RP}=1,3$; IC95\%: 1,1-1,5), doente crônico (RP = 2,2; IC95\%: 2,0$2,2)$, com cobertura por plano de saúde $(R P=1,6$; IC95\%: 1,5-1,7), com uma ou mais consultas nos últimos três meses (RP $=1,9$; IC95\%: 1,8$2,1)$, uma ou mais hospitalizações nos últimos 12 meses ( $\mathrm{RP}=1,3$; IC95\%: 1,1-1,4), autopercepção pior da saúde (RP = 1,6; IC95\%: 1,3-1,9).

Na primeira etapa do modelo hierarquizado (bloco de características sócio-econômicas) foram introduzidas as variáveis renda familiar, escolaridade e ocupação (Tabela 2). Verificouse que a renda familiar mensal maior que três salários mínimos esteve significativamente associada ao consumo de medicamentos, mesmo após ajuste pela escolaridade e ocupação (RP = 1,3; IC95\%: 1,1-1,5), confirmando os achados da análise bivariada. O mesmo não ocorreu com a escolaridade. As variáveis renda, escolaridade e ocupação foram mantidas para a análise dos blocos seguintes.

Na segunda etapa da análise de regressão logística (Tabela 2) foram introduzidas, além da renda familiar, escolaridade e ocupação, as variáveis pertencentes ao bloco de características demográficas: idade, sexo, estado conjugal e número de moradores/domicílio. O sexo feminino manteve associação positiva e estatisticamente significante com o consumo de medicamentos nos últimos 15 dias (RP = 1,4; IC95\%: 1,3-1,5). Com relação à idade, após ajuste pelas variáveis citadas acima, percebe-se que a faixa etária entre 50 e 98 anos apresentou uma queda na associação, mas permaneceu associada positivamente ao consumo de medicamentos e apresentou significância estatística $(R P=1,4$; IC95\%: 1,2-1,6). Estes resultados confirmam os achados da análise bivariada. No estado conjugal a associação existente entre casados e consumo desaparece após os ajustes pelas demais variáveis. 
Tabela 1

Prevalência do consumo de medicamentos nos últimos 15 dias conforme as variáveis estudadas.

Fortaleza, Ceará, Brasil, 2002/2003.

\begin{tabular}{|c|c|c|c|}
\hline Variáveis & $\begin{array}{l}\text { Freqüência } \\
(n=1.366)\end{array}$ & $\begin{array}{l}\text { Prevalência de consumo } \\
\text { de medicamentos }(\%)\end{array}$ & $\mathrm{p}$ valor \\
\hline \multicolumn{4}{|l|}{$\begin{array}{l}\text { Renda familiar mensal } \\
\text { (em salários mínimos }{ }^{\star} \text { ) }\end{array}$} \\
\hline Até 3 & 745 & 40,8 & $<0,01$ \\
\hline$>3$ & 621 & 55,2 & \\
\hline \multicolumn{4}{|l|}{ Escolaridade (em anos) } \\
\hline$\leq 8$ & 904 & 44,3 & $<0,05$ \\
\hline$>8$ & 462 & 52,7 & \\
\hline \multicolumn{4}{|l|}{ Ocupação** } \\
\hline Sim & 514 & 46,4 & $=0,78$ \\
\hline Não & 852 & 47,2 & \\
\hline \multicolumn{4}{|l|}{ Sexo } \\
\hline Masculino & 640 & 37,1 & $<0,01$ \\
\hline Feminino & 726 & 55,6 & \\
\hline \multicolumn{4}{|l|}{ Idade (em anos) } \\
\hline $0-15$ & 402 & 38,8 & \\
\hline $16-49$ & 704 & 44,4 & $=0,13$ \\
\hline $50-98$ & 260 & 67,2 & $<0,01$ \\
\hline \multicolumn{4}{|l|}{ Estado conjugal } \\
\hline Casado & 502 & 51,0 & $<0,05$ \\
\hline Não-casado & 864 & 44,6 & \\
\hline \multicolumn{4}{|c|}{ Número de moradores no domicílio } \\
\hline$\leq 3$ & 297 & 56,0 & $<0,05$ \\
\hline $4-5$ & 621 & 48,5 & $<0,05$ \\
\hline$\geq 6$ & 448 & 38,4 & \\
\hline \multicolumn{4}{|l|}{ Doenças crônicas } \\
\hline Sim & 227 & 88,0 & $<0,01$ \\
\hline Não & 1139 & 39,0 & \\
\hline \multicolumn{4}{|l|}{ Plano de saúde } \\
\hline Sim & 370 & 66,0 & $<0,01$ \\
\hline Não & 996 & 40,5 & \\
\hline \multicolumn{4}{|c|}{ Consulta nos últimos três meses } \\
\hline Sim & 751 & 64,5 & $<0,01$ \\
\hline Não & 615 & 34,1 & \\
\hline \multicolumn{4}{|c|}{ Hospitalização nos últimos 12 meses } \\
\hline $\operatorname{Sim}$ & 155 & 62,0 & $<0,01$ \\
\hline Não & 1211 & 45,0 & \\
\hline \multicolumn{4}{|c|}{ Autopercepção da saúde } \\
\hline Excelente/muito boa & 446 & 40,7 & \\
\hline Boa & 834 & 47,5 & $<0,05$ \\
\hline Ruim/muito ruim & 86 & 69,9 & $<0,01$ \\
\hline
\end{tabular}

* Salário mínimo $=\mathrm{R} \$ 200,00$ (outubro/2002).

** Ocupados: autônomo; funcionário público; militar; profissional liberal; proprietário de comércio,

indústria ou serviços; trabalhador em comércio, indústria ou serviços. Não ocupados: aposentado,

pensionista, desempregado, dona-de-casa, estudante, outros que não exerçam atividades. 
Tabela 2

Distribuição das variáveis de estudo de acordo com as razões de prevalência (RP)

bruta e ajustada conforme regressão logística por níveis hierárquicos e seus respectivos intervalos de confiança a 95\% (IC95\%). Fortaleza, Ceará, Brasil, 2002/2003.

\begin{tabular}{|c|c|c|}
\hline Variáveis & $\begin{array}{l}\text { RP bruta } \\
\text { (IC95\%) }\end{array}$ & $\begin{array}{l}\text { RP ajustada* } \\
\text { (IC95\%) }\end{array}$ \\
\hline \multicolumn{3}{|l|}{ 1. Bloco sócio-econômico } \\
\hline \multicolumn{3}{|c|}{ Renda familiar mensal (em salários mínimos) } \\
\hline Até 3 & 1,0 & 1,0 \\
\hline$>3$ & $1,3(1,2-1,5)$ & $1,3(1,1-1,5)$ \\
\hline \multicolumn{3}{|l|}{ Escolaridade (em anos) } \\
\hline$\leq 8$ & 1,0 & 1,0 \\
\hline$>8$ & $1,2(1,1-1,4)$ & $1,1(1,0-1,3)$ \\
\hline \multicolumn{3}{|l|}{ Ocupação } \\
\hline Sim & 1,0 & 1,0 \\
\hline Não & $1,1(0,9-1,2)$ & $1,1(1,0-1,2)$ \\
\hline \multicolumn{3}{|l|}{ 2. Bloco demográfico } \\
\hline \multicolumn{3}{|l|}{ Sexo } \\
\hline Masculino & 1,0 & 1,0 \\
\hline Feminino & $1,4(1,3-1,5)$ & $1,4(1,3-1,5)$ \\
\hline \multicolumn{3}{|l|}{ Idade (em anos) } \\
\hline $0-15$ & 1,0 & 1,0 \\
\hline $16-49$ & $1,1(0,9-1,2)$ & $0,9(0,7-1,1)$ \\
\hline $50-98$ & $1,7(1,4-1,9)$ & $1,4(1,2-1,6)$ \\
\hline \multicolumn{3}{|l|}{ Estado conjugal } \\
\hline Não-casado & 1,0 & 1,0 \\
\hline Casado/união consensual & $1,1(1,0-1,2)$ & $1,0(0,9-1,2)$ \\
\hline \multicolumn{3}{|c|}{ Número de moradores no domicílio } \\
\hline$\leq 3$ & $1,3(1,1-1,5)$ & $1,2(1,0-1,3)$ \\
\hline $4-5$ & $1,2(1,0-1,3)$ & $1,2(1,0-1,4)$ \\
\hline$\geq 6$ & 1,0 & 1,0 \\
\hline \multicolumn{3}{|l|}{ 3. Bloco saúde } \\
\hline \multicolumn{3}{|l|}{ Doenças crônicas } \\
\hline Não & 1,0 & 1,0 \\
\hline $\operatorname{Sim}$ & $2,2(2,0-2,2)$ & $2,1(1,9-2,2)$ \\
\hline \multicolumn{3}{|l|}{ Plano de saúde } \\
\hline Não & 1,0 & 1,0 \\
\hline Sim & $1,6(1,5-1,7)$ & $1,2(1,1-1,4)$ \\
\hline \multicolumn{3}{|c|}{ Consulta nos últimos três meses } \\
\hline Não & 1,0 & 1,0 \\
\hline $\operatorname{Sim}$ & $1,9(1,8-2,1)$ & $1,8(1,6-1,9)$ \\
\hline \multicolumn{3}{|c|}{ Hospitalização nos últimos 12 meses } \\
\hline Não & 1,0 & 1,0 \\
\hline $\operatorname{Sim}$ & $1,3(1,1-1,4)$ & $1,1(0,9-1,3)$ \\
\hline \multicolumn{3}{|l|}{ 4. Bloco avaliação subjetiva } \\
\hline \multicolumn{3}{|l|}{ Autopercepção da saúde } \\
\hline Excelente/muito boa & 1,0 & 1,0 \\
\hline Boa & $1,2(1,1-1,4)$ & $1,1(0,9-1,2)$ \\
\hline Ruim/muito ruim & $1,6(1,3-1,9)$ & $1,3(0,8-1,6)$ \\
\hline
\end{tabular}

* Ajustada para os fatores de confusão pertencentes a um nível hierárquico igual ou superior ao seu.
No caso do número de moradores por domicílio (Tabela 2), observou-se que, após os ajustes, as variáveis que estavam associadas positivamente (menor ou igual a três e quatro a cinco moradores por domicílio) mantiveram a associação, mas não apresentaram significância estatística. As variáveis sexo, idade e número de moradores por domicílio permaneceram no modelo.

De acordo com o modelo teórico, as variáveis do bloco saúde (doença crônica, plano de saúde, consultas nos últimos três meses e hospitalização nos últimos 12 meses) foram ajustadas para as variáveis sócio-econômicas e demográficas que permaneceram no modelo (Tabela 2). A presença de doença crônica aparece como forte preditora do consumo de medicamentos, pois verificou-se que os resultados permaneceram inalterados ( $\mathrm{RP}=2,1$; IC95\%: 1,9$2,2)$, confirmando a associação positiva e significante com o consumo de medicamentos encontrado na análise bivariada. A variável plano de saúde ajustada pelas demais perdeu força na associação, mas manteve-se associada e significante (RP = 1,2; IC95\%: 1,1-1,4), o mesmo ocorrendo com a variável realização de consultas nos últimos três meses (RP: 1,8; IC95\%: 1,61,9); já a realização de internação nos últimos 12 meses continuou associada positivamente, mas sem significância. Permaneceram para o próximo bloco: doença crônica, plano de saúde e consulta nos últimos três meses.

Na última etapa (bloco variável subjetiva) foram introduzidas todas as variáveis selecionadas nas etapas anteriores do modelo de regressão logística (Tabela 2). Observou-se que a autopercepção pior do estado de saúde após ajuste pelos possíveis confundidores perdeu a significância estatística.

\section{Discussão}

A população amostrada é representativa da população de Fortaleza, pois encontrou-se semelhanças ao comparar os dados relativos à idade, sexo, escolaridade e número de habitantes/ domicílio com os resultados do Censo Demográfico 200022.

A prevalência do consumo de medicamentos encontrada em Fortaleza $(49,7 \%)$ foi similar àquela determinada para o Nordeste do Brasil $(50,2 \%)$ no estudo realizado pelo Conselho Nacional de Secretários de Saúde (CONASS) 1. Difere, no entanto, dos valores encontrados em outros estudos realizados em diferentes cidades brasileiras $10,14,18,19,20$, onde o consumo variou de $24,7 \%$ a $70,0 \%$. Comparando-se ao con- 
sumo geral no Brasil 1, 57,0\%, o valor encontrado é inferior, mas ainda maior do que o observado em países como Espanha 13, 44,8\%, e Noruega $15,36,1 \%$.

No estudo verifica-se que renda familiar mensal, sexo, idade, presença de doença crônica, cobertura por plano de saúde e realização de consultas ao médico nos últimos três meses são prováveis fatores determinantes do consumo de medicamentos nos últimos 15 dias, em Fortaleza.

A renda familiar mensal aparece como determinante importante do consumo de medicamentos, pois observou-se que as pessoas com renda familiar superior a três salários mínimos consomem 1,3 vez mais medicamentos do que aquelas com renda igual ou inferior a três salários mínimos. Tal resultado evidencia o poder aquisitivo das pessoas como fator preditivo para o uso de medicamentos, o que também foi verificado em recentes pesquisas realizada por Bertoldi et al. 10, pelo CONASS 1 e por Fernandes 4 . Os dados demonstram que existe uma iniqüidade no acesso.

A situação é preocupante, considerando que boa parte da população brasileira depende do SUS para ter acesso aos medicamentos, que existem evidências de uma maior prevalência de doenças crônico-degenerativas nos estratos sociais mais baixos 25 e que o Estado vem falhando na distribuição gratuita de medicamentos essenciais à população 1,3,4,26. No caso específico dos pacientes carentes com doenças crônicas, o nãoacesso força o comprometimento da renda familiar ou favorece o abandono do tratamento, o que pode ocasionar agravamento do estado de saúde e conseqüente expansão dos gastos com internações e atendimentos ambulatoriais.

Teixeira 5 , em sua pesquisa sobre a adesão ao tratamento anti-hipertensivo em ambulatório de um hospital público de Fortaleza, ilustra bem a situação descrita acima. No estudo, a autora observou que dos 266 medicamentos prescritos para os 177 pacientes que realizaram consulta nesse ambulatório, só 91 (34,2\%) foram encontrados no setor público, 136 foram adquiridos no setor privado e 39 não foram adquiridos. O comprometimento da renda familiar nas classes baixas pode ser visto no estudo realizado por Fernandes 4, em Fortaleza, onde a despesa média mensal familiar com assistência à saúde nos domicílios selecionados foi de $38,5 \%$ do salário mínimo, onde, por tipo de despesa, os medicamentos tiveram a maior proporção percentual (55,5\%). A autora considerou as despesas, entre as classes D e E, proporcionalmente elevadas em relação aos demais gastos domésticos.
A escolaridade apresentou-se associada ao consumo de medicamentos, mas sem significância estatística para as pessoas com mais de oito anos de estudo. Bertoldi et al. 10 também não encontraram associação significante entre escolaridade e consumo de medicamentos em Pelotas, Rio Grande do Sul. Nos trabalhos internacionais, o grau de escolaridade aparece, principalmente, relacionado ao aspecto do consumo por automedicação, com tendência crescente de consumo entre os mais escolarizados 13,17. No Brasil, Franco et al. 21 evidenciaram a mesma coisa. De modo geral, um maior nível de escolaridade está associado a um maior conhecimento e discernimento sobre o processo saúde-doença, porém é a maior renda que propicia uma maior cobertura a planos de saúde e acesso aos serviços de saúde 2 .

Com relação ao gênero, o consumo de medicamentos foi 1,4 vez maior no sexo feminino do que no masculino. Eggen 16 encontrou resultado semelhante em Tromso, Noruega. A maior prevalência é, também, evidente em outros estudos nacionais $10,14,18,19,20$ e internacionais 12,13,15,16, e independe do tipo de consumo, com ou sem prescrição.

As mulheres em relação aos homens costumam cuidar mais de si e, historicamente, são responsáveis pelo cuidado com a saúde de suas famílias 27. Portanto, estão mais atentas à sintomatologia das doenças e costumam procurar precocemente ajuda. Ainda em relação aos homens, são as que mais utilizam os serviços de saúde 4,11,17,26. É provável que este fato esteja associado também a maiores oportunidades de atendimento nas unidades de saúde, tendo em vista os programas específicos existentes. Por outro lado, as mulheres também são submetidas a uma maior pressão da classe médica e da mídia em relação a problemas específicos de cada fase da vida, como é o caso dos incômodos do período menstrual 16, da menopausa e a sua sintomatologia associada 28 . Todos esses aspectos contribuem para que as mulheres tenham maiores chances de exposição a medicamentos.

No estudo, observa-se que o consumo aumenta com a idade e que o mesmo é $1,4 \mathrm{vez}$ maior entre as pessoas de 50 ou mais anos em comparação com as demais faixas etárias. Estudos realizados por Betoldi et al. 10, Al-Windi et al. 12, Figueiras et al. 13 e Eggen 15,16 evidenciaram um maior consumo com o aumento da idade. No estudo realizado pelo CONASS 1 , a prevalência do consumo na faixa etária de 56 anos ou mais foi de $73,3 \%$, e em estudo realizado na Espanha 13 o consumo foi maior na faixa de 60 ou mais anos $(71,1 \%)$. Esses dados acom- 
panham as evidências encontradas para essa associação.

A princípio, na análise bivariada, o número de moradores por domicílio apresentou associação significante com consumo de medicamentos, principalmente nas famílias com três ou menos pessoas, mas após os ajustes pelas variáveis de confusão perdeu a significância estatística. Estudo realizado por Loyola Filho et al. 11 constata o contrário, onde os autores verificam que existe uma tendência crescente de consumo de medicamentos sem prescrição quanto maior o número de moradores por domicílio. Apenas no trabalho realizado por Figueira et al. 13 encontra-se o maior consumo por automedicação associado entre pessoas que vivem sozinhas. Na descrição geral do consumo de medicamentos em Fortaleza existe um indicativo de maior prevalência entre os indivíduos que moram sozinhos $(62,2 \%)$.

Padecer de doença crônica foi o maior preditor do consumo de medicamentos. As pessoas com patologias crônicas consumiram 2,1 vezes mais medicamentos que os que não possuíam tais patologias. Esse é o tipo de preditor esperado, já que as pessoas nessas condições dependem de medicamentos para melhorar sua qualidade de vida. Eggen 16, em Tromso, Noruega, também encontrou associação positiva entre doentes crônicos e uso de medicamentos, onde os homens apresentavam maior consumo que as mulheres $(\mathrm{p}<0,05)$.

A cobertura por plano de saúde também foi considerada como possível fator determinante do consumo. Lima et al. 2, com base nos dados da Pesquisa Nacional por Amostra de Domicílios (PNAD) de 1998, constataram que o aumento da cobertura por planos de saúde está diretamente relacionado com a renda familiar mensal, e que existe uma associação positiva desta última com as taxas de consultas médicas, o que, sem dúvida, favorece o maior acesso aos serviços de saúde, além da indicação e aquisição de medicamentos por parte dos mais favorecidos financeiramente.

Ter se consultado nos últimos três meses apresentou associação positiva e significante com o consumo de medicamentos. As pessoas que haviam realizado alguma consulta nos últimos três meses consumiram 1,8 vez mais medicamentos que os que não realizaram nenhuma consulta. Este fato pode estar relacionado à crescente medicalização da sociedade, onde toda consulta médica tem de terminar com uma prescrição. É o que aponta Correia 26, Fernandes 4 e Eggen 16

No estudo realizado por Correia 26 em duas microrregiões do Estado do Ceará, o autor veri- ficou que 90,0\% dos usuários que se consultaram nas duas microrregiões, motivados por uma doença, receberam um tratamento. Fernandes 4 reportou em seu estudo realizado em Fortaleza, que entre as pessoas que buscaram atendimento médico, 87,9\% receberam prescrição de medicamentos ao final da consulta. Segundo Correia 26, o elevado percentual de receitas pode estar relacionado à boa qualidade da atenção em saúde, mas também pode refletir o interesse do profissional de levar a consulta a um desfecho, saltando etapas precedentes essenciais de exame do paciente e formulação de um diagnóstico. Vale lembrar que nos estudos realizados pela Prefeitura de Fortaleza, o atendimento é considerado como de baixa qualidade, tendo em vista o pouco tempo que o profissional médico dedica a seu cliente. Por trás deste fato pode estar a alta demanda de atendimento e o baixo número de recursos humanos 29,30.

Entre os autores internacionais pesquisados, Eggen 16, em Tromso, Noruega, foi o único a pesquisar essa relação, e encontrou associação positiva e significante. No estudo verificase que os homens consultam mais que as mulheres $(\mathrm{p}<0,05)$.

Apesar de o estudo ter apontado uma associação positiva, mas não estatisticamente significante entre consumo de medicamentos e ocupação, cabe observar que esse tipo de associação foi identificada por Figueiras et al. 13, na Espanha. O autor verifica que as pessoas desempregadas consomem 1,3 vez mais medicamentos que as ocupadas. O consumo entre os estudantes também é 1,2 vez maior que entre os ocupados. Mestanza \& Pamo 17, em Lima, Peru, também encontraram altas prevalências de consumo entre donas-de-casa, estudantes e desocupados, que praticavam automedicação.

No estudo, a associação entre autopercepção do estado de saúde e consumo de medicamentos perdeu sua significância quando ajustada para os fatores de confusão. Entretanto, é uma variável importante, pois as pessoas com estado pior de saúde tenderão a buscar mais assistência médica ou outras alternativas para melhorar o seu estado de saúde. Estudo realizado em Pelotas, Rio Grande do Sul, Brasil 10 constata que a prevalência de uso e número de medicamentos utilizados estão diretamente relacionados com a piora da autopercepção de saúde. Al-Windi et al. 12 evidenciaram, na Suécia, que o estado pior de saúde estava associado a um maior consumo de medicamentos prescritos pelos médicos.

Com relação a aspectos metodológicos, é importante considerar alguns fatores que po- 
dem ter limitado ou superestimado os resultados deste estudo. A época escolhida para a realização do estudo procurou eliminar ou minimizar as influências relativas a outros períodos, principalmente os relacionados à quadra chuvosa e suas conseqüências (viroses e dengue) e ao período eleitoral (eleições 2003). A não-observação desses períodos poderia superestimar o consumo de algumas classes farmacológicas. No estudo não se pode descartar, também, a influência do viés de memória, assim como de casos onde a omissão no consumo pode ter ocorrido pelo fato de a pessoa estar fazendo uso de produtos com fins ilegais ou considerados de uso íntimo. Da mesma forma, é provável que as doenças crônicas estejam subestimadas devido a problemas de memória e/ou ausência de diagnóstico entre algumas pessoas.

\section{Conclusões}

O consumo de medicamentos em Fortaleza é elevado, mas encontra-se dentro dos parâmetros observados no Brasil e no exterior. Identificou-se como possíveis fatores determinantes desse consumo a renda familiar mensal maior que três salários mínimos, o sexo feminino, a idade igual ou maior que 50 anos, a presença de doenças crônicas, a posse de planos de saú-

\section{Resumo \\ O presente trabalho tem por finalidade investigar a prevalência e os determinantes do consumo de medi- camentos pela população de Fortaleza, Ceará, Brasil. Com base em um delineamento transversal de base populacional, estudou-se uma amostra representativa de 331 domicílios, onde foram entrevistadas 1.370 pes- soas, no período de outubro/2002 a janeiro/2003. Fo- ram consideradas consumidoras de medicamentos to- das as pessoas que nos 15 dias anteriores à entrevista tenham consumido pelo menos um medicamento. Pa- ra análise estatística foram incluídas 1.366 pessoas. A prevalência do consumo de medicamentos foi de 49,7\%. Os fatores preditores do consumo na análise multivariada, por regressão logística, foram: renda fa- miliar mensal maior que três salários mínimos, idade igual ou maior que 50 anos, sexo feminino, três ou me- nos pessoas por domicílio, doente crônico, ter plano de saúde e ter se consultado uma ou mais vezes nos últi- mos três meses. A prevalência do consumo de medica- mentos em Fortaleza é elevada, mas encontra-se den- tro dos parâmetros encontrados no Brasil e no exterior. No entanto, sugere que existe uma iniqüidade no acesso aos medicamentos.}

Hábitos de Consumo de Medicamentos; Uso de Medicamentos; Farmacoepidemiologia de e a realização de consultas médicas nos últimos três meses.

O fato do acesso aos medicamentos aparecer condicionado pela renda demonstra que ele deva ser garantido pelo poder público, como determina a Constituição Federal. A não-observação desse indicador, principalmente no caso das doenças crônicas degenerativas, além de proporcionar o agravamento das enfermidades não tratadas, poderá onerar os gastos com a atenção secundária e terciária.

É importante, também, compreender que o uso de medicamentos, na grande maioria das vezes, está interligado aos problemas sociais, ambientais e ao modo de vida das pessoas; portanto, vale a pena que as autoridades governamentais e os profissionais de saúde invistam no desenvolvimento de práticas de prevenção e promoção da saúde, o que por si só, reduzirá a necessidade do uso de medicamentos pela população. Por sua vez, estas atividades deverão ser planejadas e desenvolvidas por equipe multiprofissional, assim como deve contar com a ajuda da própria comunidade, em todos os processos.

As mulheres, juntamente com os idosos e doentes crônicos, destacam-se no consumo de medicamentos, portanto, deverão ser os grupos de preferência para o delineamento de ações educativas voltadas para o uso racional de medicamentos.

\section{Colaboradores}

P. S. D. Arrais foi o responsável pela coordenação do estudo participando na concepção, desenho, coleta, organização do banco de dados, análise e interpretação dos dados e redação do artigo. H. L. L. Coelho participou na concepção e elaboração do projeto, desenvolvimento dos instrumentos, interpretação e discussão dos resultados. M. L. Barreto colaborou na análise, interpretação e discussão dos resultados. L. L. Brito colaborou na análise estatística, interpretação e discussão dos resultados. Todos os autores contribuíram na confecção do artigo.

\section{Agradecimentos}

Este trabalho é parte da Tese de Doutorado em Saúde Pública, apresentada ao Instituto de Saúde Coletiva da Universidade Federal da Bahia, em fevereiro de 2004, com o apoio da Fundação Cearense de Amparo à Pesquisa (processo n. 231/02) e da Farmácia Escola da Universidade Federal do Ceará. Agradecimentos especiais ao Dr. Francisco José Moreira Lopes, chefe da Unidade Estadual do Instituto Brasileiro de Geografia e Estatística no Ceará e às famílias entrevistadas. 


\section{Referências}

1. Conselho Nacional de Secretários de Saúde. A saúde na opinião dos brasileiros. Brasília: Conselho Nacional de Secretários de Saúde; 2003.

2. Lima JC, Azoury EB, Bastos LHCV, Coutinho MM, Pereira NN, Ferreira SCC. Desigualdades no acesso e utilização de serviços de saúde no Brasil. Saúde Debate 2002; 26:62-70.

3. Instituto Brasileiro de Defesa do Consumidor. Faltam remédios essenciais no SUS. Consumidor S.A. 2003; 69:14-9.

4. Fernandes MEP. A utilização de medicamentos na atenção à saúde, em nível domiciliar, no Município de Fortaleza [Dissertação de Mestrado]. Fortaleza: Departamento de Saúde Comunitária, Universidade Federal do Ceará; 1998.

5. Teixeira ACA. Adesão ao tratamento farmacológico da hipertensão e seus determinantes em pacientes de ambulatório [Dissertação de Mestrado]. Fortaleza: Departamento de Saúde Comunitária, Universidade Federal do Ceará; 1999.

6. Organización Mundial de la Salud. El uso racional de medicamentos. Geneva: Organización Mundial de la Salud; 1985.

7. Arrais PSD. Epidemiologia do consumo de medicamentos e eventos adversos no Município de Fortaleza [Tese de Doutorado]. Salvador: Instituto de Saúde Coletiva, Universidade Federal do Ceará; 2004.

8. Sebastião ECO. Consumo de medicamentos, um esboço dos fatores determinantes. Rev Ciên Farm 1998; 19:253-63.

9. Barros JAC. Propaganda de medicamentos: atentado à saúde? São Paulo: Editora Hucitec; 1995.

10. Bertoldi AD, Barros AJD, Hallal PC, Lima RC. Utilização de medicamentos em adultos: prevalência e determinantes individuais. Rev Saúde Pública $2004 ; 38: 228-38$.

11. Loyola Filho AI, Uchoa E, Guerra HL, Firmo JOA, Lima-Costa, MF. Prevalência e fatores associados à automedicação: resultados do projeto Bambuí. Rev Saúde Pública 2002; 36:55-62.

12. Al-Windi A, Elmfeldt D, Svärdsudd K. The relationship between age, gender, well-being and symptoms, and the use of pharmaceuticals, herbal medicines and self-care products in a Swedish municipality. Eur J Clin Pharmacol 2000; 56:311-7.

13. Figueiras A, Caamaño F, Gestal-Otero JJ. Sociodemographic factors related to self-medication in Spain. Eur J Epidemiol 2000; 16:19-26.

14. Vilarino JF, Soares IC, Silveira CM, Rodel APP, Bortoli R, Lemos RR. Perfil da automedicação em município do sul do país. Rev Saúde Pública 1998; 32:43-9.

15. Eggen AE. Patterns of medicine use in a general population (0-80 years). The influence of age, gender, diseases and place of residence on drug use in Norway. Pharmacoepidemiol Drug Saf $1997 ; 6: 179-87$
16. Eggen AE. Pattern of drug use in a general population-prevalence and predicting factors: the Tromso study. Int J Epidemiol 1994; 23:1262-72.

17. Mestanza F, Pamo O. Estudio muestral del consumo de medicamentos y automedicación en Lima metrópole. Rev Med Hered 1992; 3:101-8.

18. Simões MJS. Consumo de medicamentos e morbidade em Humaitá - Estado do Amazonas (Brasil). Medicina 1990; 23:151-8.

19. Simões MJS, Farache Filho A. Consumo de medicamentos em região do Estado de São Paulo (Brasil), 1985. Rev Saúde Pública 1988; 22:494-9.

20. Barros MBA. Saúde e classe social: um estudo sobre morbidade e consumo de medicamentos [Tese de Doutorado]. Ribeirão Preto: Faculdade de Medicina de Ribeirão Preto, Universidade de São Paulo; 1983.

21. Franco RCS, Neto JAC, Khouri MA, Nunes MO, Júnior JWS, Santana VS, et al. Consumo de medicamentos em um grupo populacional da área urbana de Salvador-BA. Rev Baiana Saúde Pública 1987 ; 13/14:113-21.

22. Instituto Brasileiro de Geografia e Estatística. Censo demográfico 2000. Características da população e dos domicílios resultados do universo. Rio de Janeiro: Instituto Brasileiro de Geografia e Estatística; 2001.

23. Conselho Municipal de Saúde de Fortaleza. Bemvindo ao século XXI. Espelho da realidade. Jornal do Conselho Municipal de Saúde de Fortaleza 2001; $1: 4$.

24. Zhang J, Yu KF. What's the relative risk? A method of correcting the odds ratio in cohort studies of cammon outcomes. JAMA 1998; 280:1690-1.

25. Lessa I, Mendonça GAS, Teixeira MT. Doenças crônicas não-transmissíveis no Brasil: dos fatores de risco ao impacto social. Bol Oficina Sanit Panam 1996; 120:389-413.

26. Correia LL. Atenção em saúde e eqüidade: uma análise de duas microrregiões assistenciais de saúde do Estado do Ceará. Rev Med Univ Fed Ceará 2000; 40:57-76.

27. Tezoquipa IH, Monreal MLA, Santiago RV. El cuidado a la salud en el ámbito doméstico: interacción social y vida cotidiana. Rev Saúde Pública 2001; 35:443-50.

28. Pedro AO, Pinto Neto AM, Costa-Paiva L, Osis MJ, Hardy E. Procura de serviço médico por mulheres climatéricas brasileiras. Rev Saúde Pública 2002; 36:484-90.

29. Prefeitura Municipal de Fortaleza. Plano municipal de assistência farmacêutica. Fortaleza: Célula de Assistência Farmacêutica, Coordenadoria de Políticas e Saúde, Secretaria Municipal de Saúde; 2003.

30. Secretaria Municipal de Desenvolvimento Social. Indicadores básicos. Boletim de Saúde de Fortaleza 2000; 4:7-15.

Submetido em 13/Ago/2004

Aprovado em 29/Mar/2005 